\title{
Study on the System for Prevention of Harmful Invasion with Access from Wireless LAN Access Point
}

\author{
Chang-Su Kim \\ PaiChai University, 155-40, Baejae-ro, SeoGu, DaeJeon, Korea \\ ddoja@pcu.ac.kr
}

\begin{abstract}
Due to a recent increase of usage of Internet and users of smart devices, there has been an increasing trend of wireless networks that can be conveniently used. However, wireless network is exposed to a weaker security than wire network. Because of such a security-related issue, users are exposed to the crime and also financial damage as well. In addition, there is an issue that using the AP of wireless network makes it feasible for a party to break into an internal network.

This study is intended to suggest a system for prevention of harmful invasion with access from wireless LAN Access Point. In order to verify the function of invasion prevention system, tools used in the penetration testing were utilized preparing for a scenario that made an attempt of attack on wireless network.
\end{abstract}

Keywords: Access Point, Intrusion detection, Intrusion Prevention, Wireless LAN, Integrated Wireless Security System

\section{Introduction}

Due to the development of wireless communication technology and an increase of use of smart device, the number of user of wireless network has been increasing. Wireless network uses radio wave transmitting and receiving data. Therefore, it fulfills network conditions requested by portable devices. In addition, it is more convenient to use wireless network than wire network that it has been an increasing trend of using wireless network. However, wireless network is exposed to a weaker security than wire network, and users of wireless network have insufficient knowledge in security that is regarded to be less important. Therefore, users tend to recklessly use wireless network. Due to such issues, users of wireless network might be exposed to a risk of crime from misuse of wireless network [1-3].

In order to solve weakness in security of wireless network, authorization technology and encryption techniques have been adopted. However, users are still exposed to the threat of security due to limitations of security technology and misuse of weakness of it. In addition, cases of crime have been continuously increasing along with an increase of use of wireless network [4].

This study is intended to detect threat of security in the wireless network environment and suggest invasion prevention system. Hence, tools used for the penetration testing were utilized in order to verify invasion prevention system while preparing for a scenario that made an attempt to attack wireless network [5].

Packets of the hacking tools were collected and analyzed. Analyzed packets were applied with rules of firewall blocking the relevant packets and IPs and preventing the system invasion. 


\section{Related Researches}

\subsection{IPS (Intrusion Prevention System)}

IPS operates based on firewall and IDS. However, it has been diversely developed in order to solve weakness of each of the systems and performing various functions. IPS flexibly provides functions including prevention and detection of invasion, and blockage of virus wall or harmful websites serving as a role of detecting or defensing from the attack with the highest level of performance. However, this system includes a separate function of coping with situations without suspension of service while minimizing the delay of network.

It prevents email worms and one way attack with Mydoom or Bagle with the technologies such as Deep Packet Inspection, Normalization, Reassemble, Pattern Matching, Packet Drop, BlockinglistRegistration. Furthermore, it blocks HTTP script infection or two-way attacks with the technology of Application Level Stateful Inspection.

Therefore, IPS is in need of enhancing efficiency of network resources by blocking harmful traffic and fulfilling following conditions[6,7].

(1) Regardless of operation of IPS, traffic shall run in the same manner and not prevent normal packet flow.

(2) It shall be operated with in-line network device that guarantees processing function beyond the maximum level of speed in the relevant network analyzing the packets and sessions in the flow of packet (data) and detecting (identifying) them to drop malicious files on a real time basis after examining them.

(3) IPS shall detect protocol/packet defined to have a possibility of causing overload in the network out of the range of signature analysis from known attacks and minimize false positive by combining many of the methodologies including the behavior-based statistics analysis for warning the abnormal traffic that are rapidly changed beyond the known traffic patterns while blocking an attempt of invasion.

(4) IPS shall provide a function of detecting and blocking attacks made in the unit of packets such as slammer worm as well as attacks in a complicated procedure.

\subsection{Analysis on the Wireless LAN Threat}

\subsubsection{Unauthorized/Illegal AP (Rogue AP)}

In general, unauthorized Aps are mostly likely connected with internal network in the company without setting a function of wireless security (ACL, WEP, 802.1x, 802.11i) for the sake of convenience of users. In such cases, it is difficult to control with existing wireless security solutions including wireless LAN switch or firewall. The range reached by wireless signal from unauthorized (rogue) AP is not limited to inside of company building. Due to this weakness, it is feasible for unauthorized client to randomly access to it and invade into the internal network of the company. Fig. 1 indicates risk factors of wireless LAN[6,8]. 


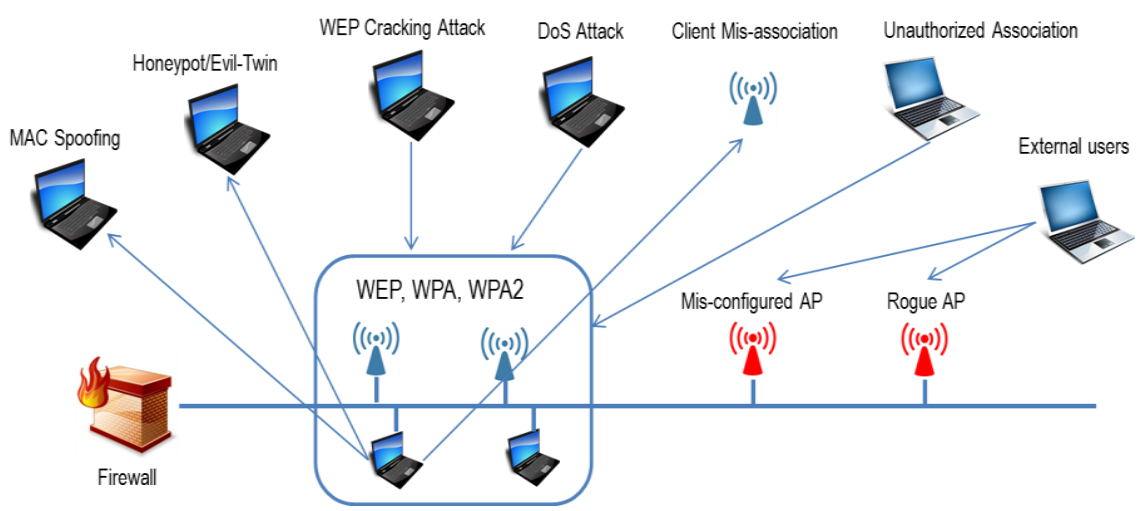

Figure 1. Risk Factors of Wireless LAN

2.2.2. Outside AP Access (Client Mis-Association): This is the case where authorized client is connected to outside unauthorized AP without approval. Aps detected around are most likely open-types without security setup, and internally authorized clients are able to disclose important resources of the company if they access to outside AP in a wireless connection.

In addition, wireless LAN is connected to most of the laptops in the No Wi-Fi environment where does not officially use wireless LAN at all. This is how a serious security threat occurs including the automatic access to outside AP when there is no AP inside.

2.2.3 Incapacitation of Availability of the Service (Dos Attack): Service avoiding attack or Dos Attack sends a particular message (De-Authentication) to all the wireless clients in the use of wireless LAN in order to hinder normal service of the system, making availability of wireless LAN service significantly deteriorated or incapacitated as the most devastated attack among hacking functions.

2.2.4 WEP Cracking Attack: There has recently been an increasing trend of using WEP key attack by utilizing the weakness of RC4-based encryption. In the past, data were collected to analyze WEP keys. However, Packet Replay and Injection technology has been developed to minimize the time required for data collection. AP is sent out to particular data packet in a repetitive manner among data transmitted to relevant Aps in order to collect replied packets.

2.2.5 Illegal Use ID for AP Service in the Company (honeypot/Evil-Twin): Wireless clients are able to co-exist in the place where multiple Aps are connected together. As for attack in the exploitation of such weakness, there are 'Honey Pot' AP and 'Evil Twin' that make an attack by configuring the unauthorized wireless network (SSID) duplicated with the wireless APs in the company. Internal wireless clients are unable to know whether SSID of AP requesting the connection is the AP from the company or illegally installed one.

\section{System Design}

Security threat occurring in the wireless network environment is difficult to be detected by a system used in wire environment such as firewall. In order to safely operate wireless network, a wireless invasion prevention system that can be integrated with a comprehensive management of continuous monitoring function is needed.

Environment configuration of the wireless invasion prevention system suggested in this study is shown in the Figure 2. 


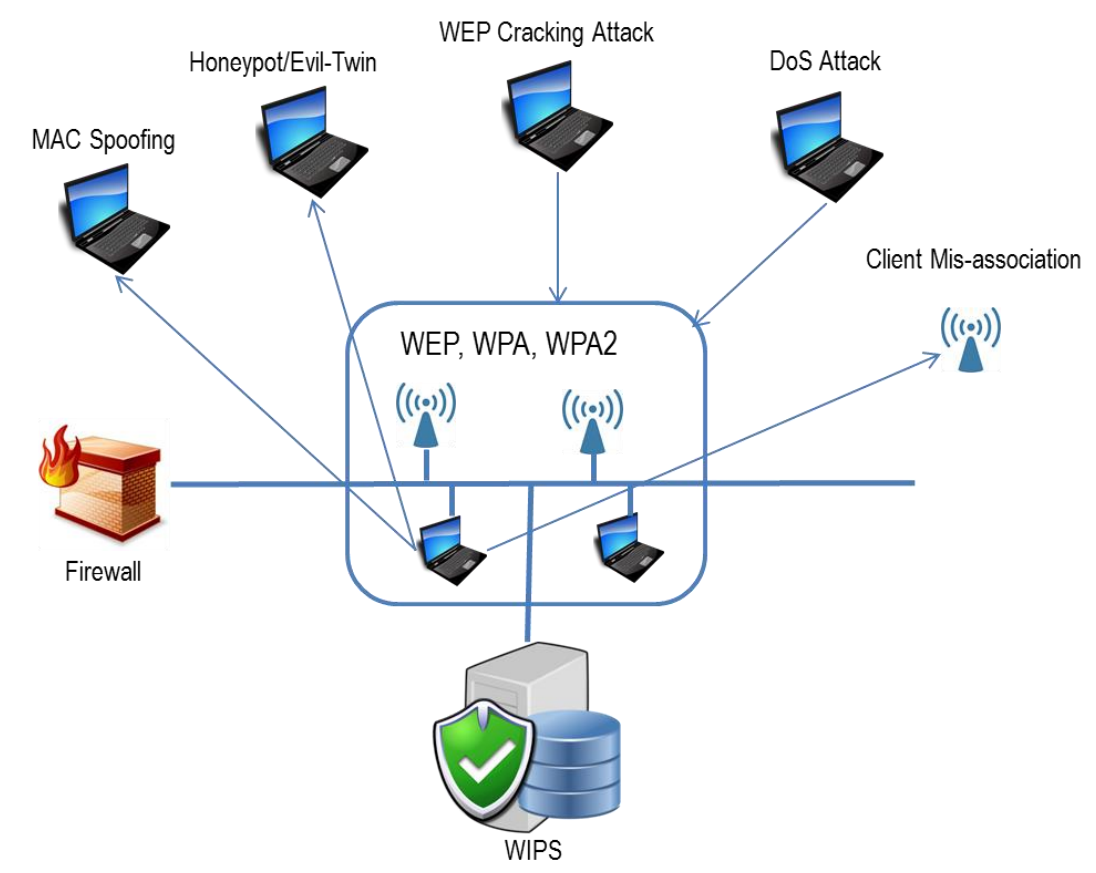

\section{Figure 2. Environment Configuration of the Wireless Invasion Prevention System}

Kismet is used in the suggested wireless invasion prevention system collecting wireless network packets and detecting invasion by examining the AP. Collected packets analyze the pattern of attack by using Wireshark. Invasion is blocked by creating a policy in firewall based on analyzed patterns. The structure of suggested wireless invasion prevention system is shown in the Figure 3.

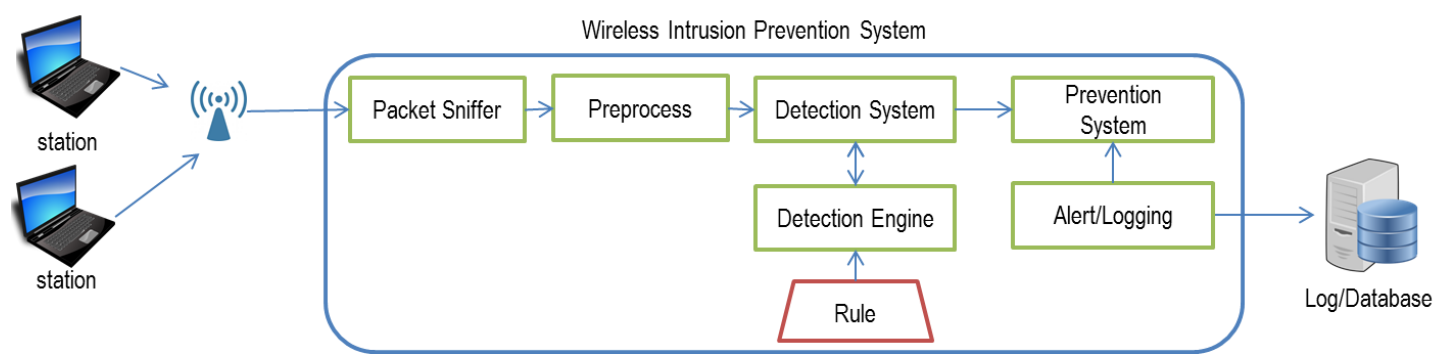

Figure 3. Structure of Suggested Wireless Invasion Prevention System

\subsection{Experiment}

Penetration testing was implemented to verify the suggested wireless invasion prevention system in this chapter stating how to detect security threat and block the invasion.

3.3.1 Experiment Environment: One PC used as a server and two laptops used as clients were utilized for the wireless invasion prevention system in the experiment environment. In addition, experiment was proceeded by using one wire router and three wireless routers.

Servers and clients were connected in the wire making it feasible to conveniently share data. In addition, clients proceed the experiment by changing three Aps in rotation. Three wireless Aps are connected with the server of wire router. Configuration of the experiment environment is shown in the Fig. 4. 


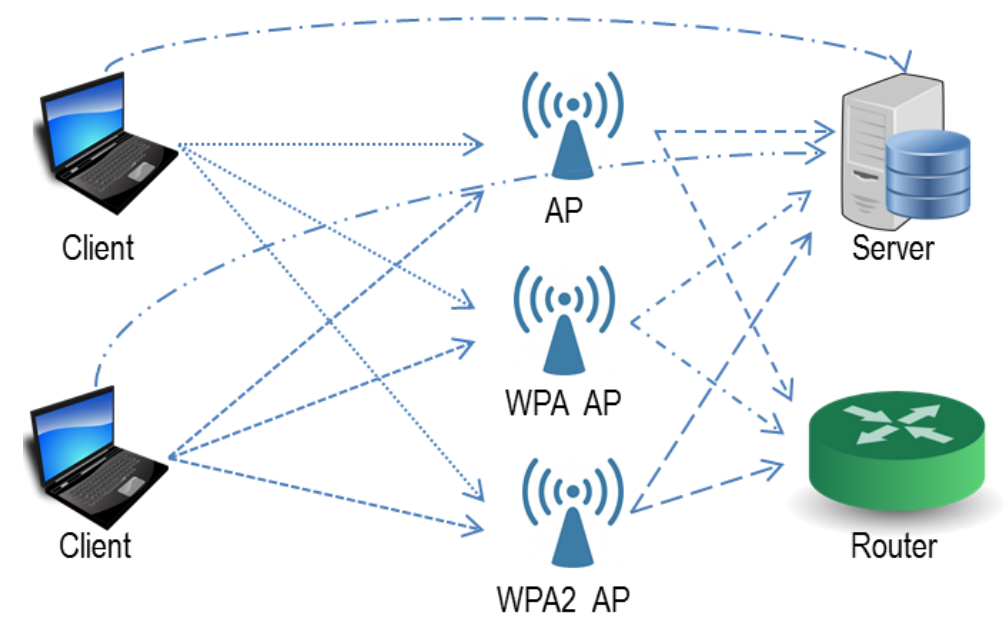

Figure 4. Configuration of the Experiment Environment

\section{1) Illegal Duplication AP Attack}

For the illegal duplication AP attack, Airmon-ng, Airodump-ng, and Airbase-ng are used proceeding the experiment. The order of illegal duplication AP attack is as follows.

(1) Create interface of the monitor mode by using Airmon-ng

(2) Collect AP information by using Airodump-ng

(3) Create AP by copying SSID value by using Airbase-ng

(4) Confirm illegally duplicated AP by using Airodump-ng

Interface in the monitor mode is created from wireless LAN card interface by using Airmon-ng. AP information is collected by using monitor mode interface created by Airmon-ng. SSID value of collected Aps is duplicated creating AP. Airodump-ng can be used to confirm the creation of illegally duplicated AP. Fig. 5 indicates illegally duplicated AP information.

\begin{tabular}{|c|c|c|c|c|c|c|c|c|c|}
\hline BSSID & PWR RXQ & Beacons & \#Data, & $\# / \mathrm{s}$ & $\mathrm{CH}$ & MB & ENC & CIPHER AUTH & ESSID \\
\hline $7 \mathrm{C}: 3 \mathrm{E}: 9 \mathrm{D}: 11: 8 \mathrm{E}: 4 \mathrm{~A}$ & -15 & 150 & 0 & 0 & 9 & 54 e & WPA & CCMP & Fake mie \\
\hline BSSID & STATION & & PWR & ate & Lo & st & Frame & es Probe & \\
\hline
\end{tabular}

Figure 5. Indicates IIlegally Duplicated AP Information

Since it is possible to confirm AP information in details as shown in the Fig. 6, illegally duplicated AP attack can be detected. 


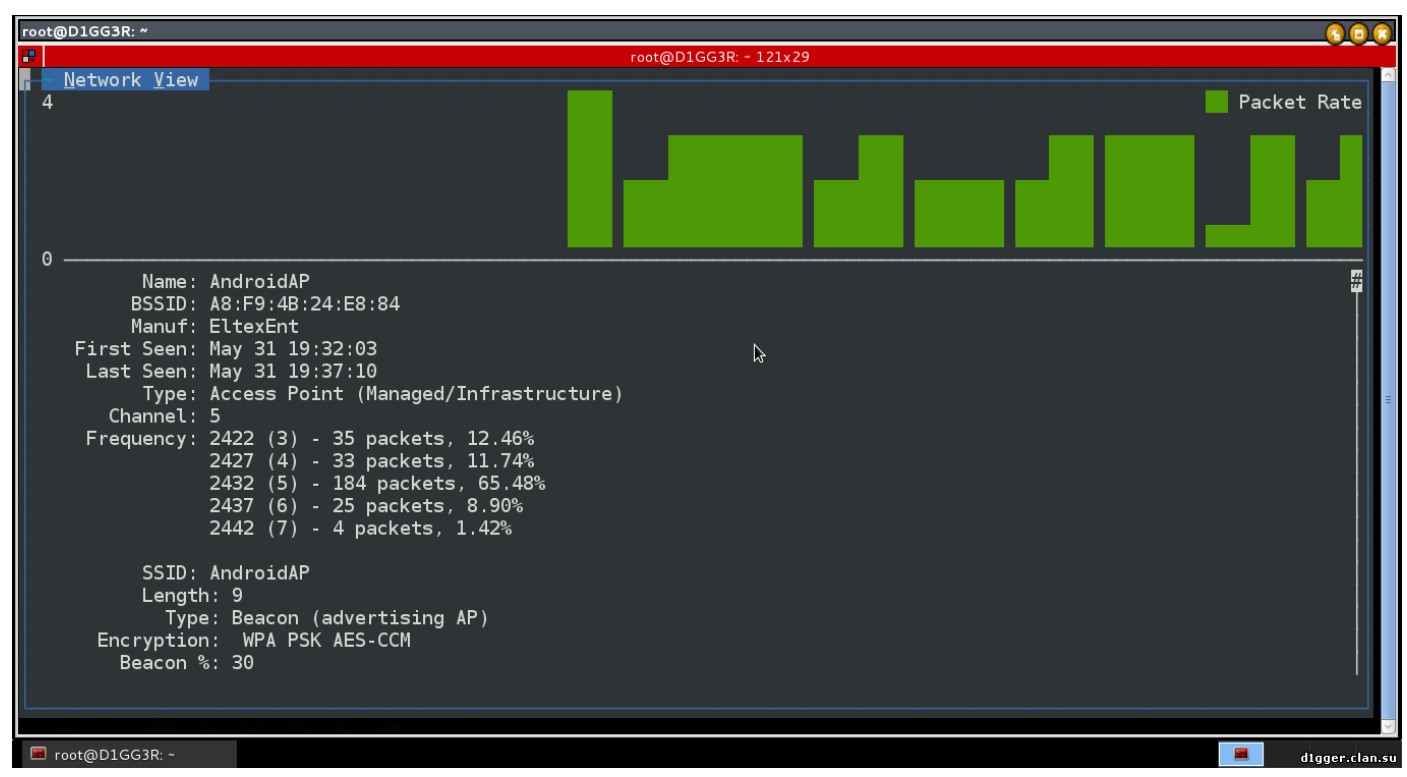

Figure 6. Confirm AP Information

\section{2) Acquisition of WPA Share Keys}

Experiment is proceeded by using Airmon-ng, Airodump-ng, Aireplay-ng, and Aircrack-ng for the WPA share key acquiring attack. The order of WPA share key acquiring attack is as follows.

(1) Create interface in the monitor mode by using Airmon-ng

(2) Collect peripheral AP information by using Airodump-ng

(3) Collect only targeted AP information by using Airodump-ng

(4) Attempt to attack of integration by using Aireplay-ng

(5) Collect WPA Handshake by using Airodump-ng

(6) Attempt to dictionary attack by using Aircrack-ng

Monitor mode interface is created by using Airmon-ng. The way of how monitor mode interface is created is in the same manner with illegally duplicated AP attack. Only the AP information for acquiring WPA share keys in the peripheral AP is collected by using Airodump-ng. Collected AP information is used making an attempt of attack for forceful reconnection.

$\mathrm{AP}$ and clients are forced to attempt a reconnection by the attack for reconnection from Aireplay-ng. Airodump-ng collects WPA Handshake in the course of reconnection. Fig. 7 is the screen of successful collection of WPA Handshake.

In the attack for acquiring WPA share keys, it is important to collect WPA Handshakes and prevent dictionary attack. In this study, packets were blocked from the attack of reconnection making AP password complicated and failing the dictionary attack. Fig. 8 indicates a screen of packet analysis from the attack of reconnection. 


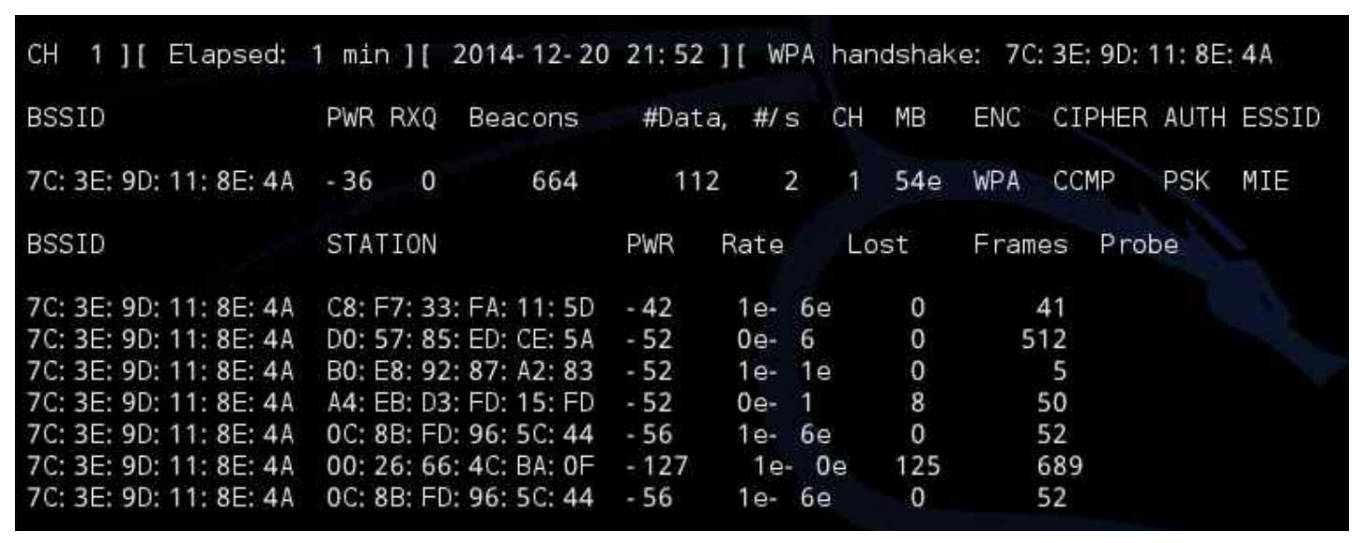

Figure 7. Screen of Successful Collection of WPA Handshake

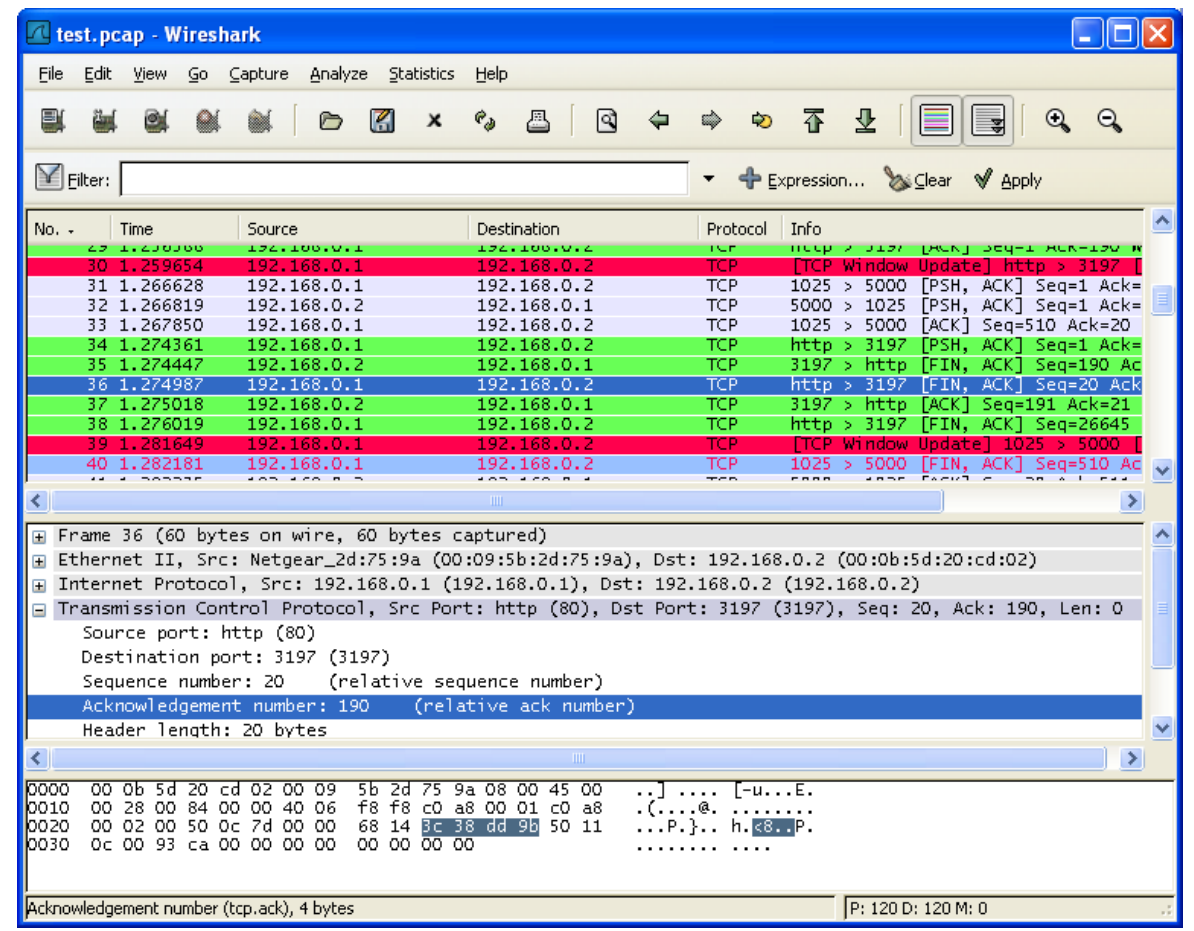

Figure 8. Screen of Packet Analysis from the Attack of Reconnection

\section{3) Man in the Middle Attack}

Experiment is proceeded for man in the middle attack by using Airmon-ng and Airbase-ng. The order of man in the middle attack is as follows.

(1) Interface in the monitor mode is created by using Airmon-ng.

(2) AP is created by using Airbase-ng.

(3) Create wire and wireless bridge

The way of how monitor mode interface and AP are created is the same manner with the acquisition of illegally duplicated AP and WPA share keys.

After creating a bridge, IP address is assigned. In the experiment, IP was not assigned on the bridge, it was not feasible for a client accessing through AP created for the man in the middle attack to use the network. Fig. 9 indicates a screen that network of the clients accessing to soft AP is not operated. 


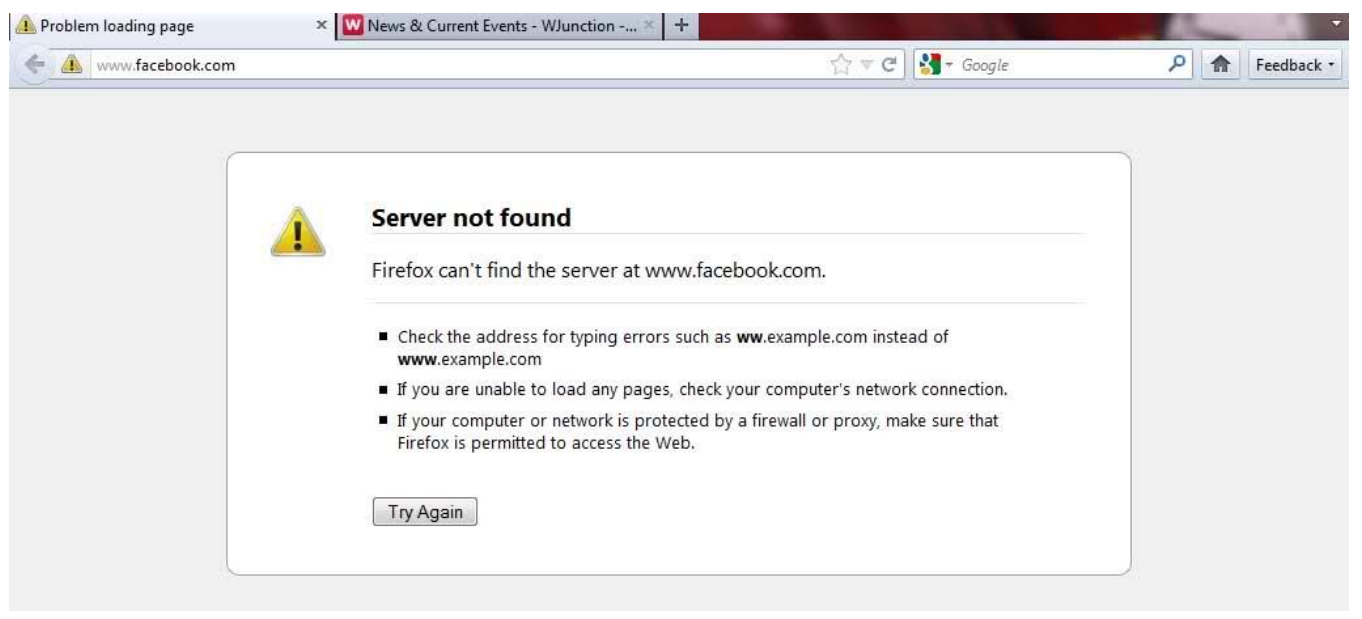

Figure 9. Network of the Clients Accessing

\section{Conclusion}

Due to an increase of wireless network, security threat occurring in the wireless network is in an increasing trend. Wireless network security technology cannot be replaced by the wire security technology. According to characteristics of wireless network, weakness in security is in more serious situations than wire network. Therefore, this study is intended to suggest a system for detecting and blocking security threat occurring in the wireless network and preventing security threat.

Wireless invasion prevention system suggested in this study collects the packets of wireless network with Drone of Kismet, and Kismet saves collected packets in a file. In addition, Kismet is used examining the wireless network environment and detecting illegally duplicated AP or unauthorized AP.

Wireshark is used to analyze packet files saved in Kismet, and packets analyzed with Wireshark are converted by a rule of firewall. Once a rule of firewall is created, it is confirmed whether invasion is blocked or not. If it is not blocked, packets are reanalyzed with Wireshark re-creating rules of firewall.

In order to verify appropriateness of wireless invasion prevention system suggested in this study, hacking tools of clients were used to proceed a penetration testing. In addition, wireless invasion prevention system was used analyzing packets of penetration testing, adding rules, and preventing invasion to verify appropriate of this study.

\section{References}

[1] J. S. Park, M. H. Park, S. H. Jung, "A Whitelist-Based Scheme for Detecting and Preventing Unauthorized AP Access Using Mobile Device" JKICS, Vol. 38, No.8, (2013), pp. 632-640.

[2] Chen, Jyh Cheng, Ming Chia Jiang, and Yi wen Liu, "Wireless LAN security and IEEE 802.11 i," Wireless Communications, (2005), pp.27-36.

[3] Shunman, Wang, et al., "WLAN and it's security problems," Proceedings of the Fourth International Conference on. IEEE, (2003), pp.241-244.

[4] Kitasuka, Teruaki, Tsuneo Nakanishi, and Akira Fukuda. "Wireless LAN based indoor positioning system WiPS and its simulation." Communications, Computers and signal Processing, Vol. 1. IEEE, (2003), pp.272-275

[5] Lefort. L, Henson. C, Taylor. K, Barnaghi. P, Compton. M, Corcho. O \& Page. K, "Semantic sensor network xg final repor,". W3C Incubator Group Report, (2013), pp. 28.

[6] Shevtsova, Ekaterina, and Christer Hansson. "Species recognition through wing interference patterns (WIPs) in Achrysocharoides Girault (Hymenoptera, Eulophidae) including two new species." ZooKeys, (2011), pp. 154.

[7] G. H. Lee, J. D. Young, "A Study on the Construction instance of the Wireless Intrusion Prevention System for illegal AP detection threat preventions", Proceedings of Symposium of the Korean Institute of communications and Information Sciences, (2008),pp. 1004-1015. 
[8] H. W. Lee, C. W. Choi, "Development of Malicious Traffic Detection and Prevention System by Embedded Module on Wireless LAN Access Point”, JKCA, Vol.6, No.12, (2006),pp. 29-39.

\section{Author}

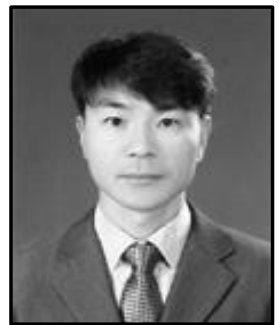

Chang Su Kim, He received his B.S., M.S., and Ph.D. degrees from the Department of Computer Engineering at Paichai University, Korea, in 1996, 1998, and 2002, respectively. From 2005 to 2012, he worked for the Department of Internet at Chungwoon University as a professor. Since 2013, he has worked in the Department of Computer Engineering at Paichai University, where he now works as a professor. His current research interests include multimedia document architecture modeling, web 2.0, and the semantic web. 
International Journal of Security and Its Applications

Vol.9, No.10 (2015) 